Article

\title{
Multi-Layer Approach for the Detection of Selective Forwarding Attacks
}

\author{
Naser Alajmi * and Khaled Elleithy * \\ Computer Science and Engineering Department, University of Bridgeport, Bridgeport, CT 06604, USA \\ * Authors to whom correspondence should be addressed; E-Mails: nalajmi@my.bridgeport.edu (N.A.); \\ elleithy@bridgeport.edu (K.E.); Tel.: +1-203-576-4703 (K.E.); Fax: +1-203-576-4766 (K.E.).
}

Academic Editor: Rongxing Lu

Received: 22 September 2015 / Accepted: 16 November 2015 / Published: 19 November 2015

\begin{abstract}
Security breaches are a major threat in wireless sensor networks (WSNs). WSNs are increasingly used due to their broad range of important applications in both military and civilian domains. WSNs are prone to several types of security attacks. Sensor nodes have limited capacities and are often deployed in dangerous locations; therefore, they are vulnerable to different types of attacks, including wormhole, sinkhole, and selective forwarding attacks. Security attacks are classified as data traffic and routing attacks. These security attacks could affect the most significant applications of WSNs, namely, military surveillance, traffic monitoring, and healthcare. Therefore, there are different approaches to detecting security attacks on the network layer in WSNs. Reliability, energy efficiency, and scalability are strong constraints on sensor nodes that affect the security of WSNs. Because sensor nodes have limited capabilities in most of these areas, selective forwarding attacks cannot be easily detected in networks. In this paper, we propose an approach to selective forwarding detection (SFD). The approach has three layers: MAC pool IDs, rule-based processing, and anomaly detection. It maintains the safety of data transmission between a source node and base station while detecting selective forwarding attacks. Furthermore, the approach is reliable, energy efficient, and scalable.
\end{abstract}

Keywords: wireless sensor networks; selective forwarding attacks 


\section{Introduction}

A sensor node is a small, lightweight sensing device. It is composed of a constrained processing unit and small amount of memory for its small operating system. Additionally, a sensor node includes a limited-range transceiver and a battery unit [1]; a mobile node also includes a mobility subsystem. WSNs often manage thousands of sensor nodes. In fact, these sensor nodes communicate with a huge number of small nodes via radio links. WSNs applications distinguish between applications based on the type of data that must be collected in the network [2]. Sensor nodes in a network gather data that are necessary to include in a smart network environment. These environments include homes, transportation systems, military installations, healthcare systems, and buildings. WSNs make it technologically possible to reorganize information and communication technology. The study of WSNs is a significant topic in computer science and engineering. It has an economic impact and affects industry.

In WSNs, sensor nodes transfer packets from the source to the base station. Because a sensor node is a limited-transmission device, it uses a multi-hop method to send packets to the base station [3]. While the communication between sensor nodes in WSNs is accomplished wirelessly by radio, adversaries can use many types of attacks. Eavesdropping, compromising nodes, interrupting or modifying packets, and injecting malicious packets compromise privacy, and denial of service attacks are threats to the security of WSNs [4]. Attackers compromise the internal sensor nodes from which they launch attacks, which are difficult to detect. A selective forwarding attack is the one of these attacks. It is an attack where a node sends some of messages to other nodes or base stations whilst dropping the sensitive information [5].

In a selective forwarding attack, malicious nodes attempt to stop the packets in a network by rejecting message forwarding. It is not easy to detect this type of attack due to the unreliability of communications. According to Karlof and Wagner [6], selective forwarding attacks can impact some routing protocols, such as TinyOS beaconing, DSR, and PSFQ. During the launch of a selective forwarding attack, a compromised node has notable consequences. A compromised node selectively drops packets. Malicious nodes work in the same manner as other nodes in the network field, but these malicious nodes try to find the important messages and drop them before sending the whole packets to the next nodes. The attackers make the sensor network rely on the redundancy forwarding by using broadcast for data to spread in the network. Based on researchers' results, limited power and low memory are obstacles that make conventional security measures inappropriate for WSNs [7]. One of the obstacles in WSNs is also energy consumption, so data transmission between sensor nodes is the major source of energy consumption and it is a serious challenge to design an energy efficient routing scheme for extending a network's lifetime [8].

\section{Related Works}

Yu and Xiao $[9,10]$ described a Lightweight Security Scheme (LWSS) as an approach that can be used to detect a selective forwarding attack in the sensor network field. LWSS uses a multi-hop acknowledgment to launch alarms by obtaining responses from the nodes that are located in the middle of a path. This approach has as a target the detection of network attackers. The target is to send an alarm that indicates a selective forwarding attack when a malicious node is discovered. The authors used two detection processes in the scheme, namely a downstream process and an upstream process. Sending an acknowledgement packet and alert packet would drain energy during the detection process. In this 
approach, a node is randomly selected as the checkpoint that sends a message acknowledging the detection of an adversary. Among the drawbacks of LWSS we may list the following:

- Resending the packet by using another route causes energy consumption and delays during the detection.

- Transmission of the acknowledgement packet and one-way key packet also cause energy consumption.

- The scheme lacks scalability.

- The scheme spends much effort on detecting the attack thus it lacks efficiency.

- The LWSS scheme could not detect the attacks under some certain conditions.

- Sending the acknowledgment causes wasted energy.

- There is no commitment to reliability if the packet is dropped.

Hai and Huh [11] described an approach to detect selective forwarding attacks. The approach is called Lightweight Detection (LWD). It consists of a lightweight mechanism where each sensor node is provided with a detection module that is built on top of an application layer. A sensor node sets its routing rules and uses information about its two-hop neighborhood to generate an alert packet. Hai and Huh suggested two routing rules to improve the monitoring system. The first rule is to determine whether the destination node forwards the packet along the path to the sink. The second rule is that the monitoring node waits and detects a packet that had been forwarded along the path to the sink. Some of the drawbacks of LWD are:

- The network has a static topology. Therefore, the LWD scheme will not detect the attack if there is a change in the type of topology.

- There is no guarantee of reliability.

- The detection scheme is not work if a node is compromised.

Deng et al. [12] proposed Secure Data Transmission (SDT) for detecting a selective forwarding attack. They used watermark technology to detect malicious nodes. Prior to employing a watermark-based technique, they used a trust value to find a source path for message forwarding. When the network is initialized, all of the nodes are assigned the same trust value. Deng et al. used a watermark-based technique to calculate the amount of packet loss. The base station compares the extracted watermark to the original watermark to detect a selective forwarding attack. Among the drawbacks of SDT Drawbacks one can mention:

- There is no data resend method if the packet is dropped.

- The SDT scheme cannot detect a malicious node if more than two.

- The scheme is not convenient for sensor-caused malicious nodes and the BS cannot compromise.

Chanatip et al. [13] proposed Received Single Strength Indicator-Extra Monitor (RSSI-EM). They used extra monitoring (EM) to eavesdrop and monitor all of the traffic when data were transferred between nodes. The value of an RSSI is that four EM nodes can be arranged to establish the positions of all of the sensor nodes, with the base station located at $(0,0)$. They assumed that the attackers could capture and damage the nodes. Therefore, all of the sensor nodes must protect themselves or be made from tamper-resistant hardware. The RSSI-EM drawbacks are: 
- The topology is static thus any change will affect the efficiency of the scheme.

- The scheme accuracy is low.

\section{Problem Identification}

A selective forwarding attack is difficult to detect in a network. The adversary installs a malicious node in the network area, which drops packets. Once the malicious node is present in the network, it organizes routing loops that attract or refuse network traffic. Additionally, malicious nodes can perform some activities that impact the network. These activities include extending or shortening source routes, generating false messages, and attempting to drop significant messages (Figure 1). Packet drops are common due to environmental conditions, but it is also possible that an attacker can simply drop a packet purposefully [14]. Packets that are dropped selectively sometimes come from one node or a group of nodes. The malicious node refuses to forward the packets. In addition, the base station does not receive the entire message. There is a need for a new paradigm for detecting selective forwarding attacks that can increase the detection rate while consuming less energy.

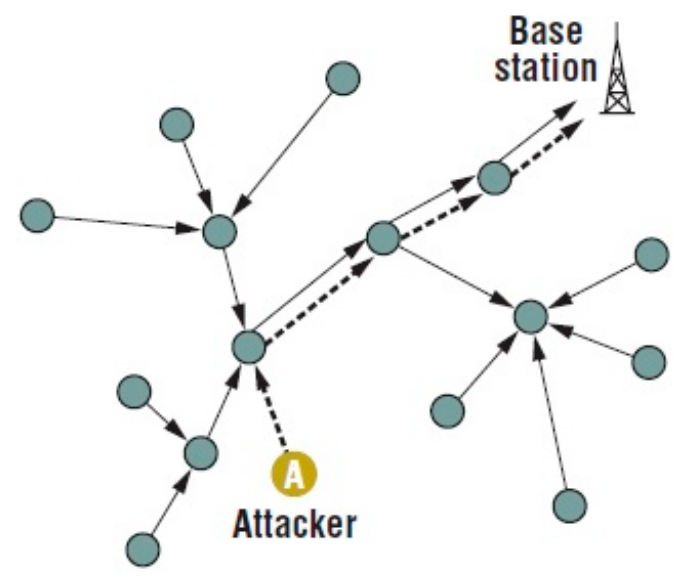

Figure 1. Selective Forwarding Attacks-Redrawn [15].

\section{Proposed System}

Sensor networks are vulnerable to many types of security attack. A malicious node tries to create blocks that occur while messages are being transferred between sensor nodes in the network by, for instance, forwarding a message along another path, generating an inaccurate network route, and delaying the transfer of packets between nodes. With a limited radio communication range, wireless sensor nodes communicate with each other by a multi-hop path [16]. In a sensor network area, data are sent to the base station through routers. An attacker compromises the nodes by attacking the network resources. Selective forwarding attacks destroy the packets transmitted between the source and base station. For this purpose, a malicious node refuses to transfer the whole packet, attempting to drop considerable data and therefore, the whole packet is not transferred to the base station. Furthermore, physical attacks frequently occur in WSNs because they are easy for adversaries to execute. Selective forwarding attacks can seriously impact the data collection of WSNs and data will be lost with compromised sensor nodes [17]. Selective forwarding detection (SFD) discovers a secure route for data to be sent from one node to other 
nodes. In this section of the paper, we introduce the assumptions and a multi-layer approach for detecting selective forwarding attacks.

\subsection{Assumptions}

To detect selective forwarding attacks within certain applications we must make some assumptions. We assume that all nodes have the same specifications. All nodes in the network have the same energy at the starting point and maximum energy. As well as, we assume that nodes are uniformly distributed in network in a random manner. Malicious nodes should not drop any packets before launching a selective forwarding attack, and an adversary cannot attack nodes during their deployment. Nodes can send data to a base station. Received Signal Strength Indicator-RSSI is the mechanism to measure the distance between the base station and a node.

\subsection{Selective Forwarding Detection (SFD) Using Multi-Layers}

Rule-based IDS, also known as signature-based IDS, is one of the mechanisms for protecting a network from security threats. The network layer in WSNs is threatened with many types of attacks, including wormhole and sinkhole attacks. Our proposal focuses on the selective forwarding attack. We design a multi-layer approach to detection that includes the three security layers shown in Figure 2. Figure 3 shows the details of the algorithm. The first layer is a pool of MAC IDs. In this layer, the important information is filtered and stored. The information includes message fields (e.g., packet, destination, and source IDs) that are useful for rule-based processing. The second layer is the rule-based processing layer. In this layer, there are some rules that must be applied to the stored data. Incoming traffic is either accepted or rejected. In addition, no rules are applied to a message that fails. The third layer is the anomaly detection layer, which detects the false negative anomalies that comprise unknown attacks. The second layer (rule-based processing) and the third layer (anomaly detection-based IDS) can identify and control selective forwarding attacks in all phases. The three layers are supported by three algorithms. These algorithms are used to resolve the attacks on the network. The detection approach saves energy by using little time and memory. It chooses a secure route along which to transfer data between the source and base station. Furthermore, the SFD approach will be reliable, energy efficient, and scalable. All of these factors are important for sensor node networks. Additionally, this approach has a high accuracy rate. We compared our approach with other approaches and found SFD has a 98.3\% accuracy rate so it is higher than others.

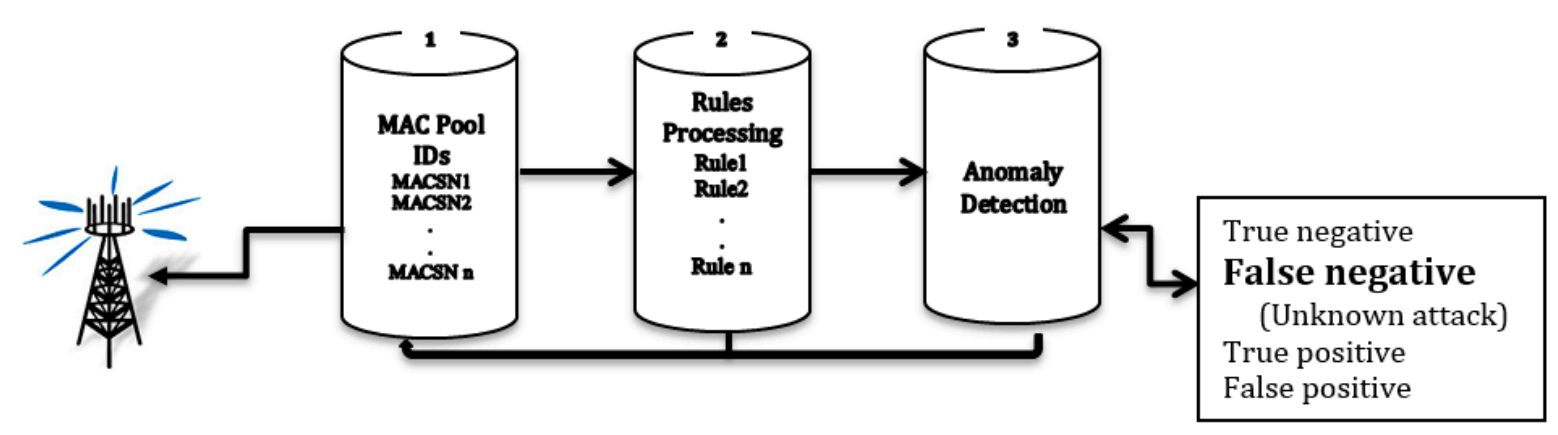

Figure 2. Multi-Layers in Rules-Based IDS. 


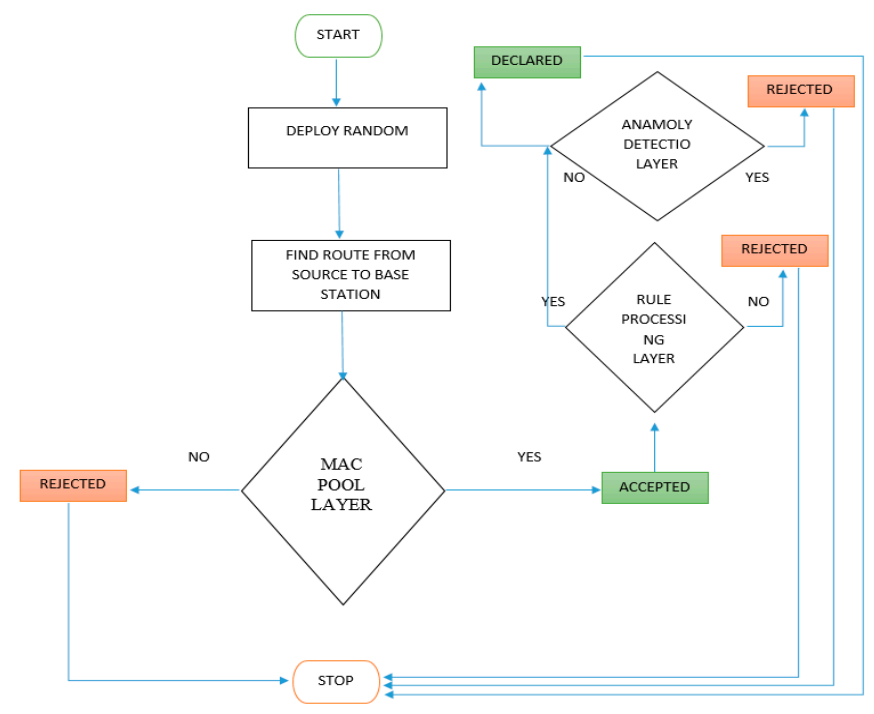

Figure 3. Selective Forwarding Attack Detection Flowchart.

\subsection{Selective Forwarding Detection (SFD) Algorithms}

\subsubsection{MAC Pool of IDs Layer}

The first layer consists of a pool of MAC IDs that filters and matches the traffic. Each traffic packet is monitored. The packet is matched to identify malicious activity using message fields (e.g., the packet, destination, and source IDs). It checks whether a node is legitimate or malicious. If a node is assigned a value of zero, it drops a packet and is considered malicious. Otherwise, it is accepted as a legitimate node. In our study, we analyze the malicious nodes that are detected in the first step using an algorithm based on the pool of MAC IDs as shown in Algorithm 1.

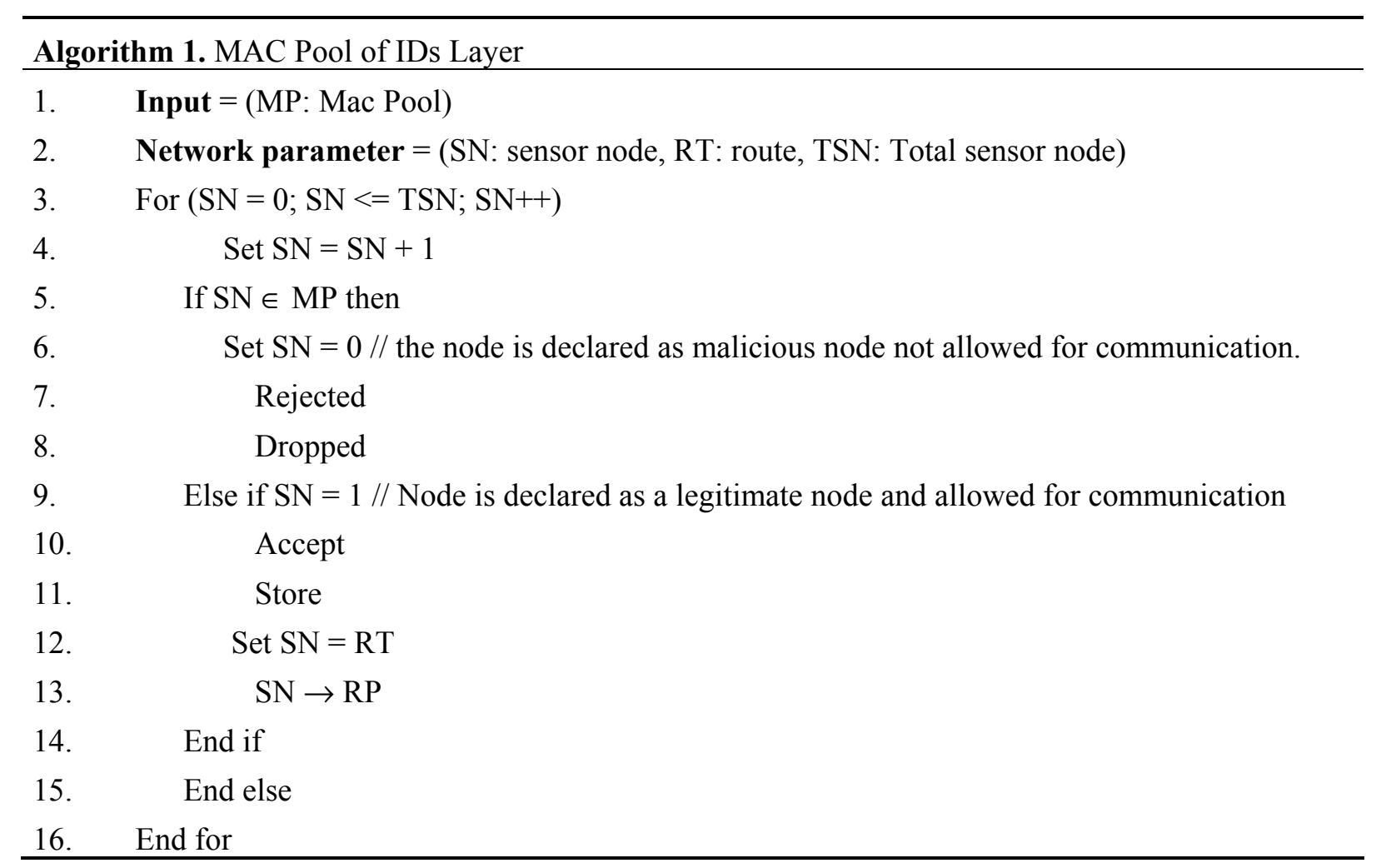




\subsubsection{Rules Processing Layer}

The second layer involves rule-based processing. It is the middle layer. It detects known attacks using rules. These are techniques used to define and describe the normal operations for detecting selective forwarding attacks. Rules must be applied before nodes are deployed in a network area. The rule-based processing layer checks the traffic by comparing it to a list of rules. If the traffic satisfies at least $90 \%$ of the rules, the node is confirmed to be legitimate (Algorithm 2). Therefore, the traffic will be returned to the pool of MAC IDs for release. If the traffic does not satisfy $90 \%$ of the rules, the node is considered doubtful and is rejected. Details of the rules are given in Table 1.

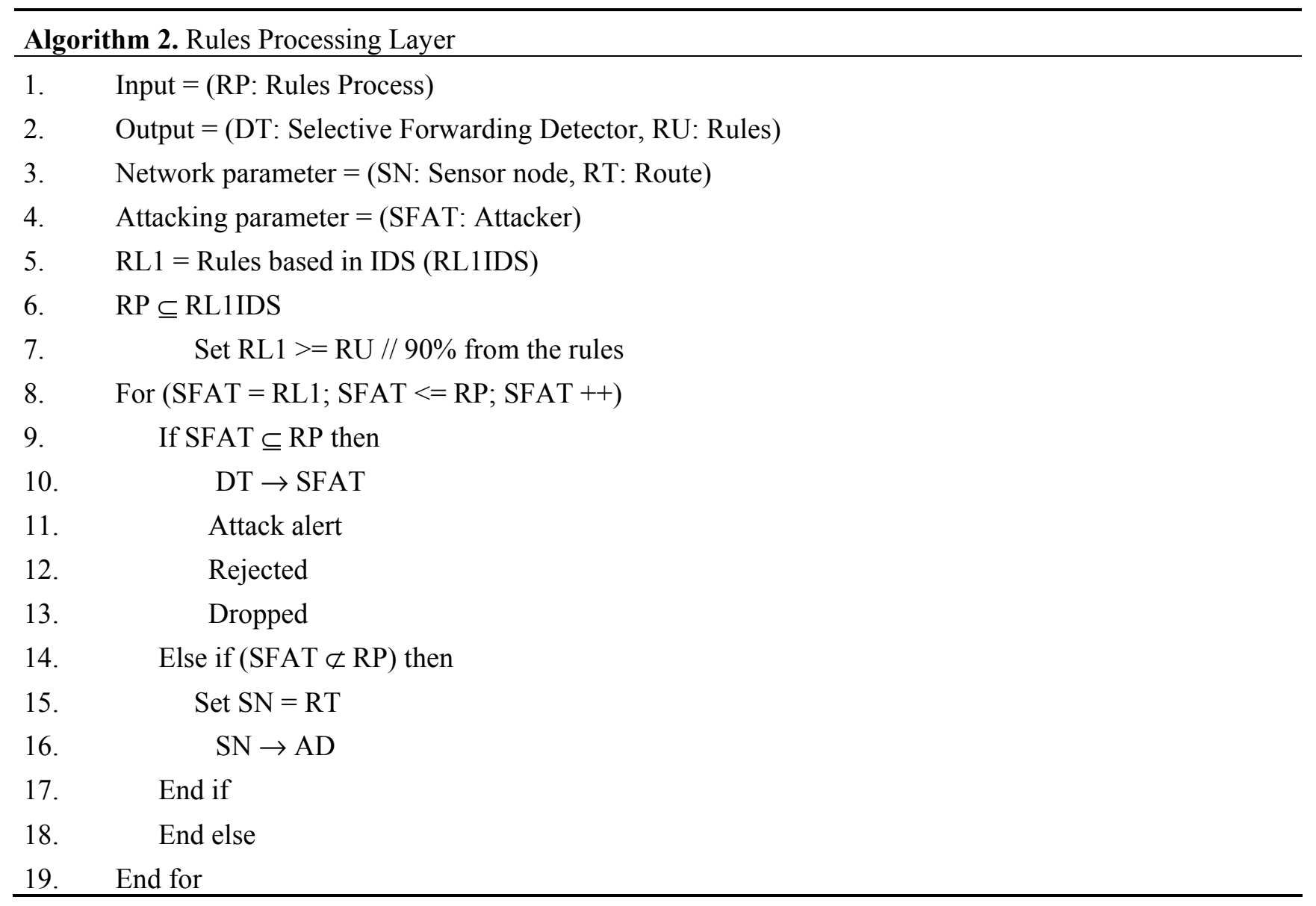

Table 1. Rules based in selective forwarding attack.

\begin{tabular}{|c|c|}
\hline Rule No. & Rule Description \\
\hline Rule1 & Each node wait to see if the neighbor node forward the message or not. \\
\hline Rule2 & $\begin{array}{l}\text { The node that will receives message has to checks the transfer's identity to make sure it is not } \\
\text { change during transferring. }\end{array}$ \\
\hline Rule3 & Each node makes sure that the next node has a shared key for negotiation. \\
\hline Rule4 & Each node has a message route when it wants to transfer to other node. \\
\hline Rule5 & Each sensor node must have ACKs. \\
\hline Rule6 & Each sensor node must have the same ACK that use. \\
\hline Rule7 & Each node has not created a new response before the previous one transfer. \\
\hline Rule8 & Each node has to send the message using the correct route. \\
\hline Rule9 & Each sensor node only communicates with other sensor nodes that locate in the same topology. \\
\hline
\end{tabular}




\subsubsection{Anomaly Detection Layer Based on Intrusion Detection System}

The third layer involves anomaly detection, which is the recognition of unknown attacks. This layer checks the traffic that comes from the rule-based processing layer. Therefore, it works to analyze the traffic. The possible results of anomaly detection are false negative, false positive, true negative, and true positive. If the algorithm determines that an unknown attack is a false negative, it sends an alert and rejects the relevant packet. Otherwise, the traffic is returned to the pool of MAC IDs by confirming the legitimacy of the node as shown in Algorithm 3.

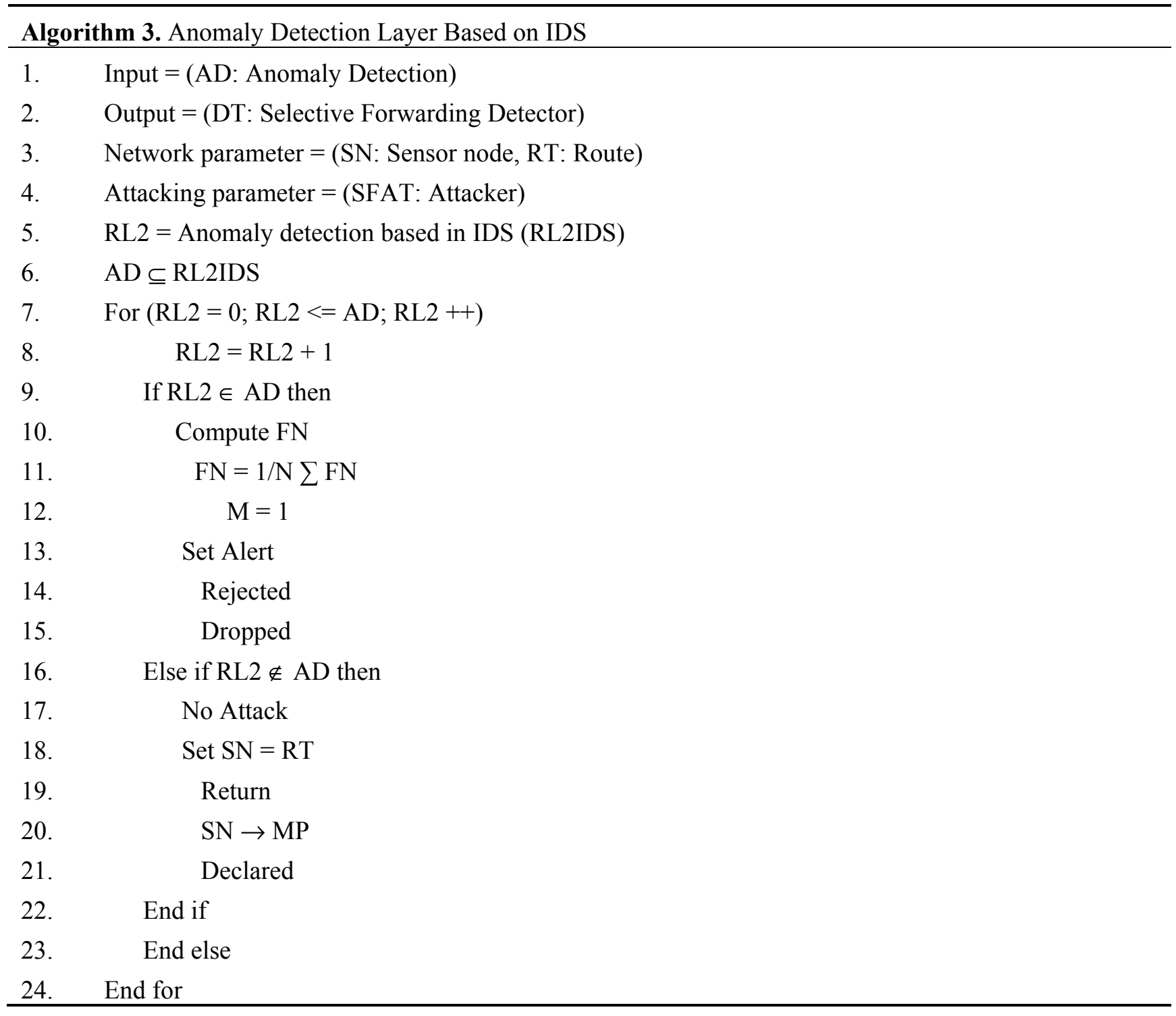

\section{Reliable, Energy Efficient and Scalable (RES) Model}

The goal of a reliable, energy-efficient and scalable (RES) model is to extend the network lifetime while maintaining the Quality of Service (QoS). The network lifetime is the most significant metric of wireless sensor networks. RES also aims to balance the energy utilization for unevenly distributed sensor nodes to provide longer secure surveillance for military bases. In the military base surveillance, there is a high probability that nodes will die by forwarding heavy traffic. 
In order to develop reliable communication, we have to determine a reliable path from the sender node to the base station, as the $\forall K$ number of the sensor nodes in the reliable optimal $R P$ path is given as:

$$
\prod_{i=0}^{\forall K} R P_{i j}
$$

Let us assume that WSNs are perceived as the 2D graph with vertex $V$ and edges $E$ written as $G(V, E)$ with transmission range $T_{r}$ so that the maximum reliable communication can be obtained using BellmanFord algorithm's link measurement properties $B F$ given as:

$$
B F=\frac{\theta \sigma_{y}^{2}}{T_{r} d_{x}{ }^{-n}}
$$

Once, we start searching the reliable path for communication then we can this write as:

$$
R P: R P_{\max } \prod_{(i, j)}^{N} R P_{i j}-R P_{\min } \sum_{(i, j \in R P)}^{N}-\log \frac{1}{R P_{i j}}
$$

Once we are able to find the reliable communication pathway, then we have to balance the energy consumption. We define the network lifetime when the sensor node first time drains its energy. Ideally, prolonging the network lifetime requires satisfying the following conditions: total consumed energy for all sensor nodes in the network, the differences between the node's individual energy consumption, average energy consumption of each sensor node, and energy consumed for transmitting the packet and for receiving the packet.

Total consumed energy for all sensor nodes in the network should be considered as minimal $\prod \Delta E_{m}$. Determining the differences between the node's individual energy consumption $\Delta E_{m}\left(1 \leq k \leq S_{n}\right)$ and an average energy consumption $\Delta E_{a}$ is the minimal energy. The differences can be accumulated as:

$$
\rho^{2}=\sum_{k=0}^{n} k\left(\Delta E_{m}-\Delta E_{a}\right)^{2}
$$

where $\rho^{2}$ is differences between minimal energy and an average energy of the sensor node.

After determining the differences, we focuses on an average energy $\Delta E_{a}$ consumption of each sensor node that can be written as:

$$
\Delta E_{a}=\sum_{k=0}^{n} k\left(\Delta E_{m}\right)
$$

As well as, we need to determine the number of generated packets generated by sensor node $k$ :

$$
\omega_{p}=\left(\Delta \beta_{t} \prod_{u \in S(k)}^{n} Y_{u k}-\Delta \gamma_{r} \prod_{v \in S(k)}^{n} Z_{v k}\right)
$$

Once a node joins and leaves the network, the communication performance is affected and the provision of QoS is degraded. We address scalability in our design to overcome the performance degradation.

Let us consider the number of joining nodes $k_{j}$ in the network. The size of the network is limited and it does not accept a load of more than $k_{j} \leq 1 \leq k_{t}$. Given that the network will accept $k_{l}$ sensor nodes in the network, thus, scalable probability of network can be defined as: 


$$
S_{p}{ }^{+}=\sum_{k=0}^{\infty}\left(k_{t}\right)+k_{j} \times \iint_{i=0 \& j=0}^{N+}(\Delta p)^{n}+(\nabla \mathrm{p})
$$

where $(\Delta p)^{n}$ the number of delivered packets from the sensor nodes that are already part of the network and $\nabla \mathrm{p}$ the number of packets delivered by nodes joining the network and $S_{p}{ }^{+}$the scalable probability when sensor nodes join the networks.

\section{Results and Discussion}

The approach to detecting selective forwarding attacks is tested using a simulation. In the simulation, 200 sensor nodes are deployed in a network with an area of $800 \times 800 \mathrm{~m}^{2}$ using NS2 (Table 2). Therefore, each node had a transmission range of $35 \mathrm{~m}$ and a sensing range of $30 \mathrm{~m}$. The energetic cost of a node is $5 \mathrm{~J}$, and there are 180 static and 20 mobile nodes. We calculated the amount of energy consumed. Figure $4 \mathrm{a}$ describes the reliable detection rate of our approach and other works. The reliable detection rate is important to extend the network lifetime. We proved the number of packets successfully received at the destination node. It clearly shows that SFD is stable at almost the same level when the time increased from $0 \mathrm{~min}$ to $27 \mathrm{~min}$. The reliable detection rate is $98.4 \%$. The reliable detection rate for the LWSS, LWD, SDT, and RSSI-EM approaches are not stable and go down when the time increases. The reliability rates are $88.2 \%, 90.6 \%, 89.6 \%$, and $86.3 \%$, respectively. Energy is also an important factor. Figure $4 \mathrm{~b}$ shows the energy consumption performance of the LWSS, LWD, SDT, and RSSI-EM approaches with 180 static nodes and 20 mobility nodes. In comparing our proposed SFD approach with the other approaches, we assume the $10 \%$ of nodes are malicious and $10 \%$ of the nodes are mobile. As a result, we saw different percentages of energy consumption for each one of these approaches, which consumed $75.1 \%, 81.8 \%, 69.1 \%$, and $68.5 \%$, respectively. Thus, the total of malicious nodes and energy consumption appears. Figure $4 \mathrm{c}$ shows the probability detection of selective forwarding attacks and other competing schemes with 50\% malicious nodes and static nodes. As a result, SFD has a high probability of almost $96 \%$. In Figure $4 d$ we show the packet delivery ratio with $50 \%$ malicious nodes and $25 \%$ mobile nodes. Between 5\% and 10\% malicious nodes, the SFD approach has a ratio of $99.2 \%$, higher than the values of the other approaches which $94.4 \%, 94.1 \%, 94.3 \%$, and $94.2 \%$. The accuracy rate of SFD and other competing selective forwarding mechanisms are shown in Figure 4e. The accuracy of our approach is more than $98 \%$. The network consumes less energy when it includes mobile nodes; therefore, it was $60.4 \%$ at the highest point, and the energy cost was low. If there are malicious nodes along the routes, the SFD approach is able to reduce the communication overhead. The new approach is more effective while the detection of nodes is increased.

Table 2. Experiment Parameters.

\begin{tabular}{cc}
\hline Parameters & Description \\
\hline Transmission Range & $35 \mathrm{~m}$ \\
Sensing Range of node & $30 \mathrm{~m}$ \\
Initial energy of a node & $5 \mathrm{~J}$ \\
Bandwidth of node & $60 \mathrm{~Kb} / \mathrm{Sec}$ \\
Number of legitimate sensors & 120 \\
\hline
\end{tabular}


Table 2. Cont.

\begin{tabular}{cc}
\hline Parameters & Description \\
\hline Number of Malicious nodes & 80 \\
Size of network & $800 \times 800 \mathrm{~m}^{2}$ \\
Buffering capacity & 45 Packets buffering capacity at each node \\
Data Packet size & 128 bytes \\
Simulation time & $27 \mathrm{~min}$ \\
Tx energy & $15.2 \mathrm{~mW}$ \\
Rx energy & $11.8 \mathrm{~mW}$ \\
Power Intensity & $-18 \mathrm{dBm}$ to $13 \mathrm{dBm}$. \\
\hline
\end{tabular}

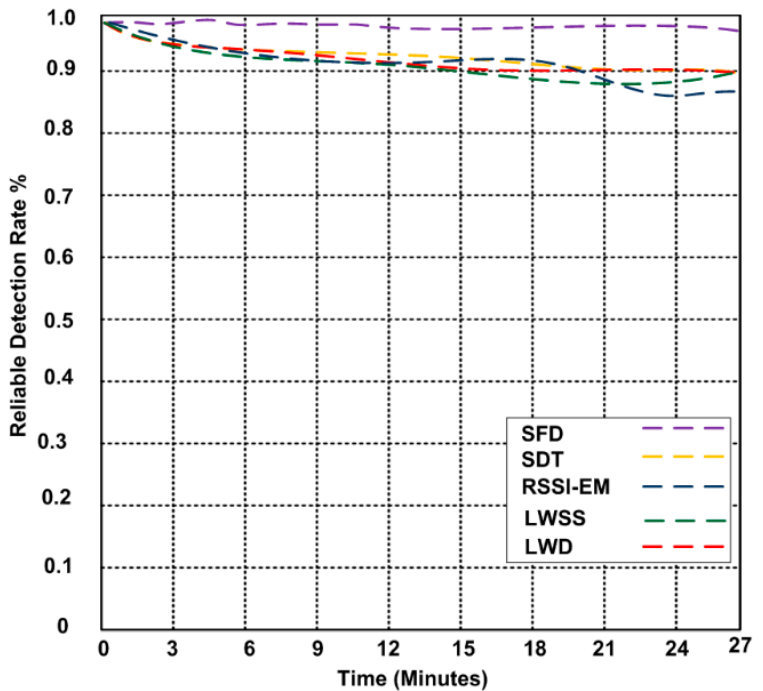

(a)

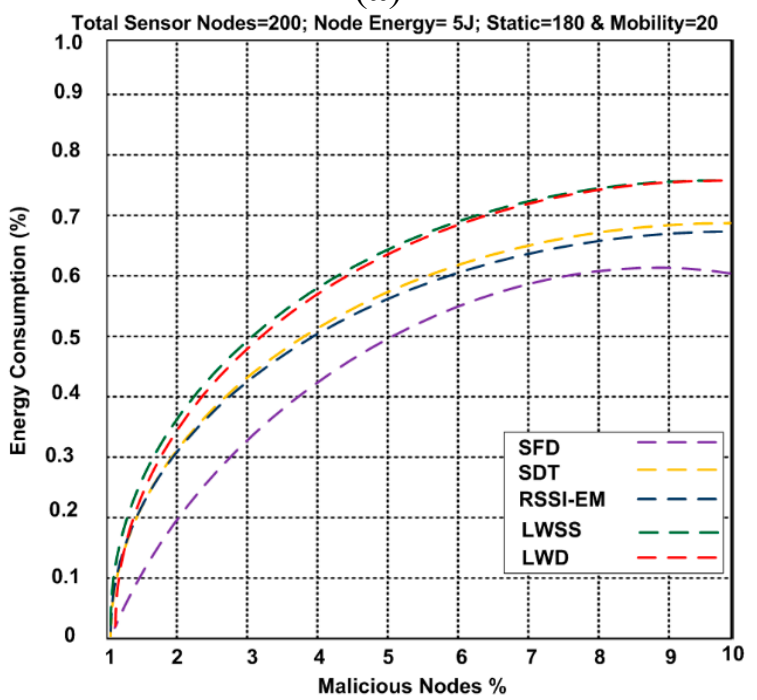

(b)

Figure 4. Cont. 


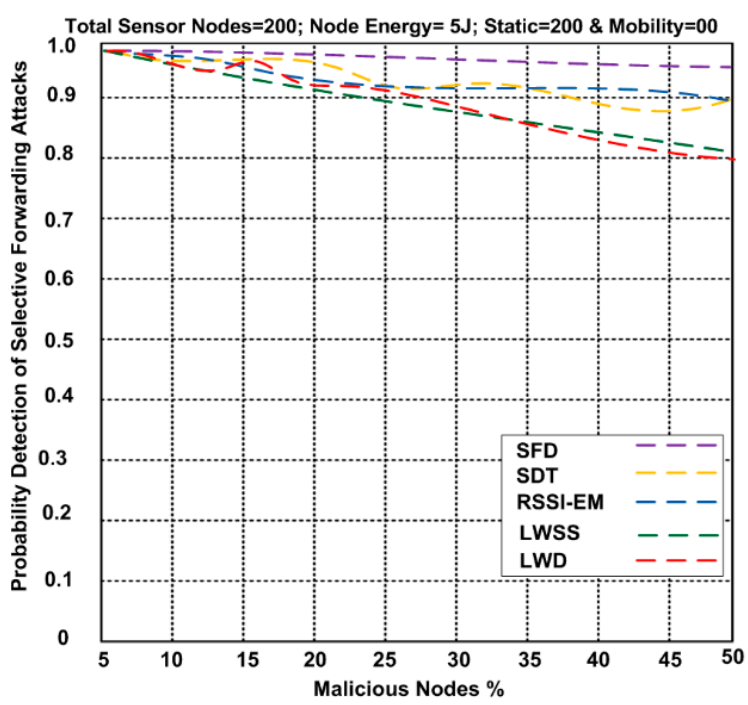

(c)

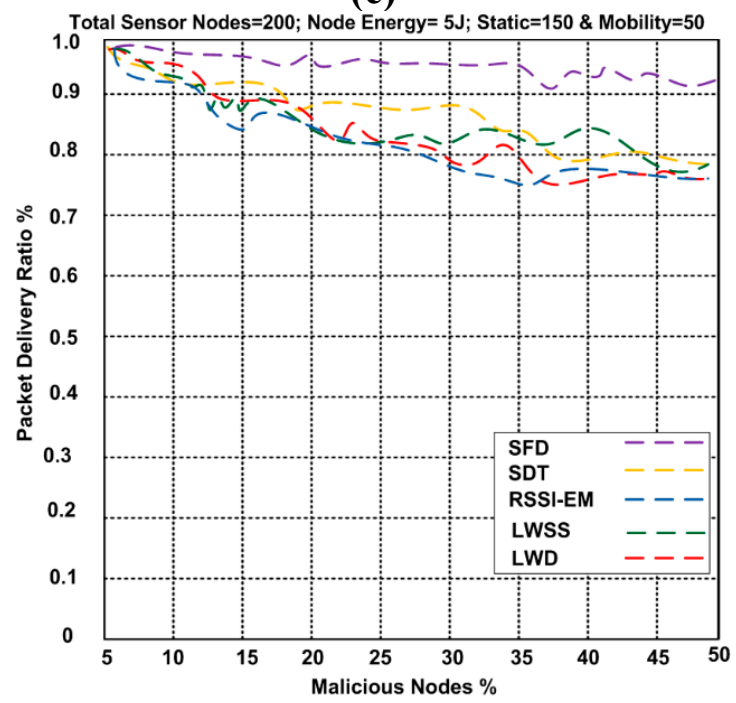

(d)

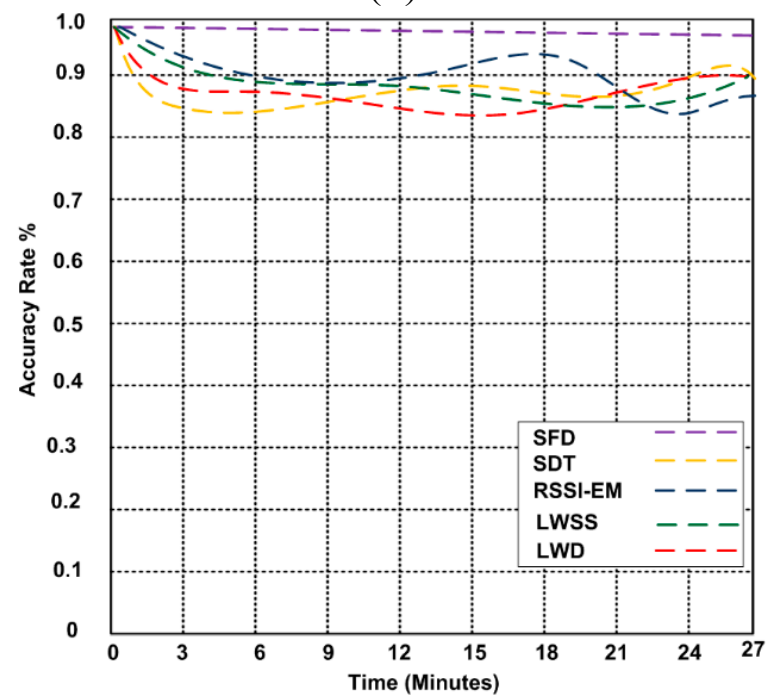

(e)

Figure 4. (a) Reliable detection rate of selective forwarding attack; (b) Energy consumptions; (c) Probability detection of selective forwarding attack; (d) Packet delivery ratio; (e) Accuracy rate. 


\section{Conclusions}

Security, reliability, energy efficiency, and scalability are challenging design issues for wireless sensor networks. We present in this work a new approach, called Selective Forwarding Detection (SFD), to detect one type of severe attack, selective forwarding attacks. This type of attack severaly affects the communication network of nodes by breaking the communication links. It is a multi-layer detection approach. The multi-layer detection framework consists of three layers, each of which is supported by a different algorithm. In the first layer, we used an algorithm based on a pool of MAC IDs that authenticates the incoming traffic to determine whether a node is legitimate or malicious. In the second layer, we used a rule-based processing algorithm, which checks the traffic by comparing it to a list of rules. In the third layer, we used an anomaly detection algorithm to identify unknown attacks, which appear as false negatives, send an alert, and reject the traffic. In addition, the framework was validated using NS2. Based on the simulation results, we demonstrated that this approach's detection rate and energy consumption are better than other approaches, therefore, the FD approach is a reliable, energy efficient, and scalable technique to prevent forwarding attacks.

\section{Author Contributions}

This work is done as a part of Naser Alajmi Ph.D. dissertation under the supervision of Khaled Elleithy. Both authors equally contributed to this work.

\section{Conflicts of Interest}

The authors declare no conflict of interest.

\section{References}

1. Akyildiz, I.; Su, W.; Sankarasubramaniam, Y.; Cayirci, E. Wireless sensor networks. Comput. Netw. 2002, 38, 393-422.

2. Mamun, Q. A Qualitative Comparison of Different Logical Topologies for Wireless Sensor Networks. Sensors 2012, 12, 14887-14913.

3. Pathan, A.-S.K.; Lee, H.-W.; Hong, C.S. Security in Wireless Sensor Networks: Issues and Challenges. In Proceedings of the 8th International Conference Advanced Communication Technology, Dublin, Ireland, 20-22 February 2006; Volume 2, pp. 1043-1048.

4. Perrig, A.; Stankovic, J.; Wagner, D. Security in Wireless Sensor Networks. Commun. ACM 2004, 47, 53-57.

5. Kaplantzis, S.; Shilton, A.; Mani, N.; Sekercioglu, Y.A. Detecting Selective Forwarding Attacks in Wireless Sensor Networks using Support Vector Machines. In Proceedings of the 3rd International Conference on Intelligent Sensors, Sensor Networks and Information, Melbourne, Australia, 3-6 December 2007; pp. 335-340.

6. Karlof, C.; Wagner, D. Secure routing in wireless sensor networks: Attacks and countermeasures. Ad Hoc Netw. 2003, 1, 293-315. 
7. Martins, D.; Guyennet, H. Wireless Sensor Network Attacks and Security Mechanisms: A Short Survey. In Proceedings of the 13th International Conference on Network-Based Information Systems (NBiS), Gifu, Japan, 14-16 September 2010; Volume 1, pp. 313-320.

8. Wang, S.-S.; Chen, Z.-P. LCM: A link-aware clustering mechanism for energy-efficient routing in wireless sensor networks. IEEE Sens. J. 2013, 13, 728-736.

9. Yu, B.; Xiao, B. Detecting Selective Forwarding Attacks in Wireless Sensor Networks. In Proceedings of the 20th International Parallel and Distributed Processing Symposiun, Rhodes Island, Greece, 25-29 April 2006; pp.1-8.

10. Xiao, B.; Yu, B.; Gao, C. CHEMAS: Identify Suspect Nodes in Selective Forwarding Attacks. J. Parallel Distrib. Comput. 2007, 67, 1218-1230.

11. Hai, T.H.; Huh, E.-N. Detecting Selective Forwarding Attacks in Wireless Sensor Networks Using Two-Hops Neighbor Knowledge. In Proceedings of the Seventh IEEE International Symposium on Network Computing and Applications, Cambridge, MA, USA, 10-12 July 2008; pp. 325-331.

12. Deng, H.; Sun, X.; Wang, B.; Cao, Y. Selective Forwarding Attack Detection using Watermark in Wireless Sensor Networks. In Proceedings of the International Colloquium on Computing, Communications Control, and Management, Sanya, China, 8-9 August 2009; pp. 109-113.

13. Tumrongwittayapak, C.; Varakulsiripunth, R. Detecting Sinkhole Attack and Selective Forwarding Attack in Wireless Sensor Networks. In Proceedings of the 7th International Conference on Information, Communications and Signal Processing, Macau, China, 8-10 December 2009; pp. 1-5.

14. Geetha, V.; Chandrasekaran, K. Enhanced Beta Trust Model for Identifying Insider Attacks in Wireless Sensor Networks. Int. J. Comput. Sci. Netw. Secur. 2013, 13, 14-19.

15. Sridevi, K.J. Message Authentication in Sensor Networks Using En-Route Filtering. Int. J. Adv. Netw. Appl. 2015, 6, 127-131.

16. Duan, J.Q.; Yang, D.; Zhu, H.Q.; Zhang, S.D.; Zhao, J. TSRF: A Trust-Aware Secure Routing Framework in Wireless Sensor Networks. Int. J. Distrib. Sens. Netw. 2014, 2014, doi:10.1155/2014/209436.

17. Cui, B.; Yang, S.J. NRE: Suppress Selective Forwarding Attacks in Wireless Sensor Networks. In Proceedings of the IEEE Conference on Communications and Network Security, San Francisco, CA, USA, 29-31 October 2014; pp. 229-237.

(C) 2015 by the authors; licensee MDPI, Basel, Switzerland. This article is an open access article distributed under the terms and conditions of the Creative Commons Attribution license (http://creativecommons.org/licenses/by/4.0/). 